\section{Endogenous inhibitors of angiogenesis}

\section{Christiana Ruhrberg}

Endothelial Cell Biology, Imperial Cancer Research Fund, PO Box 123, 44 Lincoln's Inn Fields, London WC2A 3PX, UK

(e-mail: ruhrberg@icrf.icnet.uk)

Journal of Cell Science 114, 3215-3216 (2001) (C) The Company of Biologists Ltd

In the course of embryonic development, during some normal physiological processes in the adult, such as the female reproductive cycle, and also in a variety of pathologies, including tumour growth, new blood vessels develop from the pre-existing vascular network through endothelial cell sprouting, proliferation and fusion - a process termed angiogenesis (Risau, 1997). Blocking tumour angiogenesis has become a promising approach in managing cancer: in recent years, a large number of anti-angiogenic drugs and recombinant proteins have entered clinical trials, and some are already in the final phase of testing (see http://cancertrials.nci.nih.gov).
Research into tumour angiogenesis was kick-started by the realisation that tumours are not able to grow beyond a size of $1-2 \mathrm{~mm}^{3}$ without first recruiting their own vascular supply to deliver oxygen and nutrients (Folkman, 1971). A wide variety of pro-angiogenic molecules were subsequently found to be released from cancerous cells, most importantly vascular endothelial growth factor (VEGF) and basic fibroblast growth factor (bFGF; step I; Kerbel, 2000).

Generally, angiogenesis can be inhibited at any of a number of key steps in the endothelial growth cycle, in which a quiescent vessel becomes an actively growing and invading endothelial tube (Kerbel, 2000). The activation of resting vessels by tumour-derived angiogenic factors such as VEGF can be blocked by, for example, neutralising antibodies or compounds that impair downstream signalling (inhibition of step I). Degradation of the extracellular matrix by activated endothelial cells can also be prevented, which suppresses sprouting and invasion of growing capillaries into their surrounds (inhibition of step II).
Alternatively, endothelial cell proliferation can be inhibited by agents that block signalling within the cell and arrest its division cycle (inhibition of step III) or by agents that prevent the maturation of nascent endothelial cells into functional tubes (inhibition of step IV). Finally, endothelial cells can be forced to apoptose, which destroys existing tumour vessels and thereby impairs survival of large tumour masses and metastasis through the vascular route (inhibition of step V). Excitingly, some inhibitors target the tumour vasculature at more than one of these steps, which should increase their efficiency. For example, in addition to stimulating resting vessels, VEGF promotes both proliferation and survival of endothelial cells, and antibodies against this endothelial cell-specific growth factor might therefore target both quiescent and actively cycling endothelial cells.

Hijacking naturally occurring inhibitors of angiogenesis to block tumour vascularisation might minimise side effects commonly associated with the use of artificial compounds in cancer

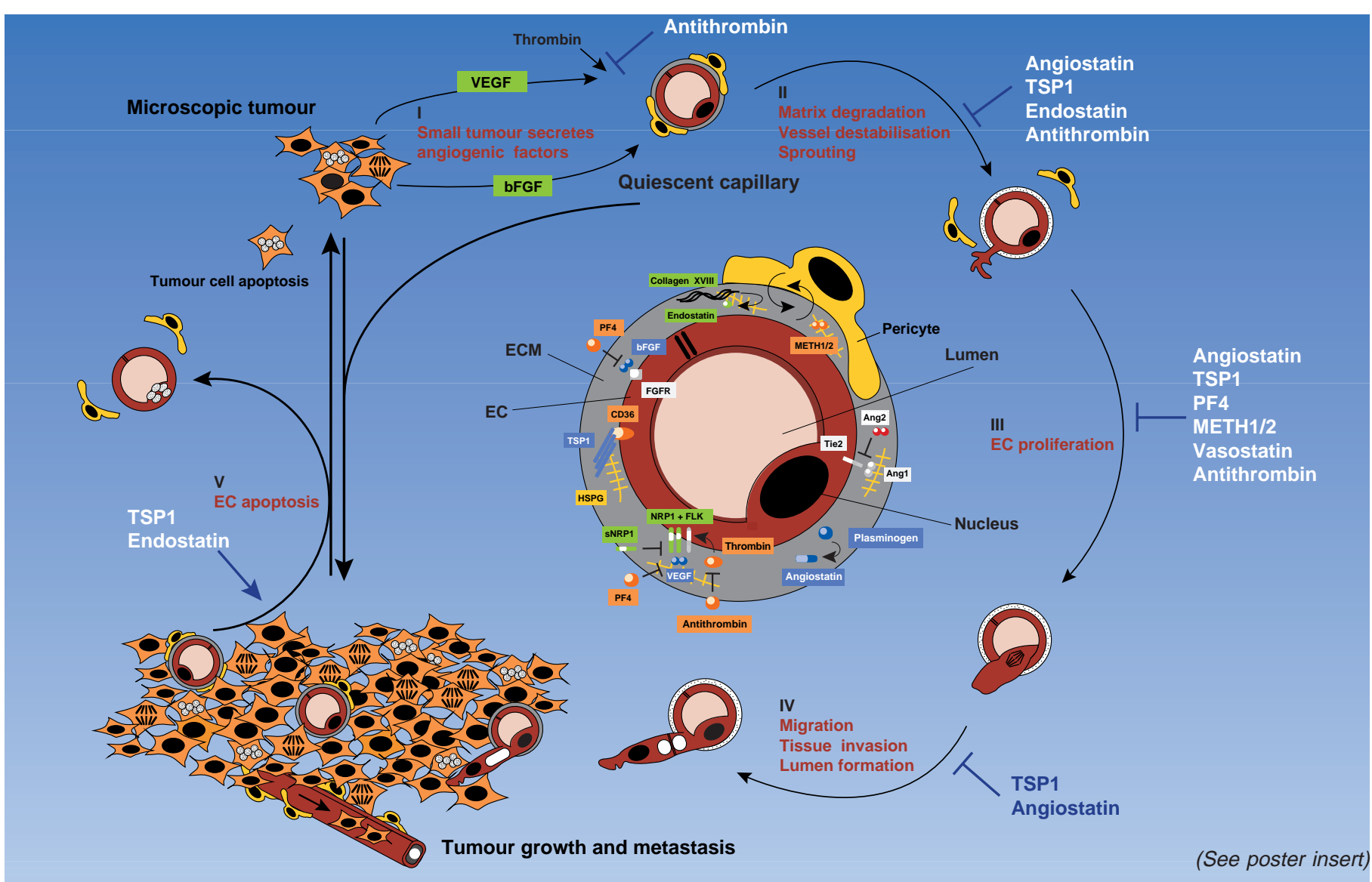


therapy. Experimental tests on tumour bearing animals are indeed promising: recombinant angiogenic inhibitors such as endostatin can shrink tumours to a bearable size and might be used to keep them small indefinitely, since resistance does not build up to these agents, and their toxicity is low. If these are used in combination with more traditional cancer drugs, which target tumour cells directly, cancers that are currently incurable might at least become manageable.

Abbreviations/definitions: Ang1/2, angiopoetin 1/2 - ligand for Tie-2 receptor; angiostatin - kringledomain containing fragment of plasminogen (a protein involved in the blood clotting/fibrinolytic pathway); anti-thrombin - serpin-family member involved in the blood clotting/fibrinolytic pathway; endostatin - fragment of type XVIII collagen in the extracellular matrix; bFGF, basic fibroblast growth factor; FGFR, FGF receptor; FLK-1, fms-like kinase 1; VEGF receptor (also known as VEGFR2 or KDR); HSPG, heparan sulphate proteoglycan in the extracellular matrix; Meth1/2, protein containing metalloproteases and thrombospondin domains; PF4, fragment of platelet factor 4; NRP1, neuropilin 1 - FLK-1 co-receptor; sNRP1, secreted fragment of neuropilin 1; Tie-2, angiopoetin receptor (also known as Tek); thrombin - protein in the blood clotting/fibrinolytic pathway that upregulates VEGF receptors; TSP1, thrombospondin 1 - extracellular matrix protein; VEGF, vascular endothelial growth factor; vasostatin - fragment of calreticulin (a protein in the endoplasmic reticulum involved in calcium signalling).

\section{References}

Risau, W. (1997). Mechanisms of angiogenesis. Nature 386, 671-674.

Folkman, J. (1971). Tumour angiogenesis: therapeutic implications. New Engl. J. Med. 285 , 1182-1186.

Kerbel, R. S. (2000). Tumour angiogenesis: past, present and the near future. Carcinogenesis $\mathbf{2 1}$ 505-515.

\section{Cell Science at a Glance on the Web}

Electronic copies of the poster insert are available in the online version of this article at jcs.biologists.org. JPEG and PDF files (see supplemental material) can be downloaded for printing or use as slides.

\section{Year 2001 \\ Travelling Fellowships}

JCS offers fellowships of up to US\$4000 to graduate students and post-docs wishing to make collaborative visits to other laboratories. These are designed to cover the cost of travel and other expenses, and there is no restriction on nationality. Applicants should be working in the field of cell biology and intend to visit a laboratory in another country. Each application is judged on the excellence of the candidate, and the importance and innovative quality of the work to be done.

Application forms can be downloaded from our Web site at http://jcs.biologists.org. Please send the completed application form, together with a copy of your CV, an account of the work to be done and a breakdown of the costs involved, as well as detters of recommendation from the heads of the laboratory in which you currently work and the laboratory you hope to visit, to the Production Editor at the address below.

Journal of Cell Science Editorial Office,

The Company of Biologists Limited,

Bidder Building, 140 Cowley Road, Cambridge CB4 ODL, UK 